\title{
Numerical and experimental assessment of the modal curvature method for damage detection in plate structures
}

\author{
Francesco Mosti, Giuseppe Quaranta, and Walter Lacarbonara ${ }^{\mathrm{a}}$ \\ Department of Structural and Geotechnical Engineering, Sapienza University of Rome, via Eudossiana 18, 00184 Rome, \\ Italy
}

\begin{abstract}
This paper is concerned with the use of numerically obtained modal curvatures for damage detection in both isotropic and composite laminated plates. Numerical simulations are carried out by using COMSOL Multiphysics as FEM solver of the governing equations, in which a Mindlin-Reissner plate model is assumed and defects are introduced as localized smoothed variations of the baseline (healthy) configuration. Experiments are also performed on steel and aluminum plates using scanning laser vibrometry. This study confirms that the central difference method greatly amplifies the measurement errors and its application leads to ineffective predictions for damage detection, even after denoising. As a consequence, different numerical techniques should be explored to allow the use of numerically obtained modal curvatures for structural health monitoring. Herein, the SavitzkyGolay filter (or least-square smoothing filter) is considered for the numerical differentiation of noisy data. Our results show that this filter is effective in identifying the damaged regions of plate structures.
\end{abstract}

\section{Introduction}

Vibration-based damage detection techniques have an advantage associated with their global approach by which faults within a mechanical system can be identified without a priori information about their location and regardless of their accessibility. Moreover, automatic real-time vibration-based structural health monitoring (SHM) systems can be effectively implemented. In this context, approaches exploiting changes in the modal characteristics of the structure caused by damages are especially attractive. While the validity of modal damping as damage index is still a controversial topic, variations in natural frequencies and mode shapes have been largely exploited to determine the occurrence of damage. In particular, changes in mode shapes yield local information which makes them more sensitive for damage localization, provided that a sufficient number of measurement points is acquired. However, several studies have shown that modal displacements are not very sensitive to faults. On the contrary, changes in the modal curvatures with respect to those of the baseline (healthy) structure lead to a more effective damage index. In [1] it was observed that modal curvatures exhibit a peak at the damage location identified as the position where a spike is manifested in the function obtained by subtracting the modal curvature of the undamaged structure from that of the damaged situation. This evidence inspired the definition of a number of damage indices (see, e.g., [2]). Most of the current applications are based on the extraction of modal displacements from dynamic measurements, and thus the curvatures have to be estimated numerically. The differentiation of noisy data is well known to be an illposed problem, and thus the practical usefulness of modal curvatures obtained by numerical techniques can be heavily compromised. As a consequence, some recent attempts

\footnotetext{
a e-mail: walter.1acarbonara@uniroma1.it
}

have addressed this issue by proposing novel approaches [3-6].

The present contribution is part of a larger effort aimed at assessing the effectiveness of damage detection by modal curvatures as well as at improving their reliability for practical SHM applications. This paper is particularly concerned with the use of modal curvatures for damage detection in plate structures. Within this framework, damage detection of composite laminated plates based on modal curvatures has been discussed in [7]. Longitudinal modal curvatures were calculated using the central difference method, and dynamic measurements were performed by using a scanning laser vibrometer (SLV). Curvatures obtained from measured modal displacements were also recently considered in [8] to identify defects in composite T-stiffened panels. In the present paper, an extensive numerical study is conducted for isotropic and composite laminated plates. Simulations are performed using COMSOL Multiphysics. A Mindlin-Reissner plate model is assumed and defects are introduced as localized smoothed variations of the baseline configuration. Experiments are also performed on steel and aluminum plates using SLV. Although the central difference method is largely preferred to calculate the modal curvatures, our study confirms that it greatly amplifies the measurement errors. Its final outcomes are ineffective for damage detection, even if the measurement errors are as low as possible and denoising is performed. On the contrary, numerical and experimental investigations demonstrate that the Savitzky-Golay filter yields reliable predictions for practical SHM applications.

\section{Damage detection by modal curvatures}

We assume that noisy modal displacements $\phi_{i}$ are available at positions $\left(x_{i}, y_{i}\right)$, and the measurement points along a given $y$ co-ordinate are taken to be spaced by the con- 
stant sampling distance $\Delta x$. The problem is concerned with the determination of the second derivative with respect to $x$ denoted by $\phi^{\prime \prime}\left(x_{i}\right)$, i.e, a suitable estimation of the exact modal curvature at $x_{i}$ about direction $y$. Therefore, the 2D numerical differentiation problem is reduced to multiple 1D problems, as it was done in previous researches [7]. In this paper, two numerical differentiation techniques are considered, namely, the standard central difference approximation and the Savitzky-Golay filter. The methods described below apply to all targeted mode shapes and plate configurations (undamaged or damaged). Any reference to the mode number and to the beam configuration is, therefore, omitted for sake of conciseness.

The most popular approach for extracting curvatures via numerical differentiation from (displacement) mode shapes $\phi$ is based on the central difference approximation. For the $i$ th point, this leads to the following expression:

$$
\phi^{\prime \prime}\left(x_{i}\right)=\frac{\phi_{i-1}-2 \phi_{i}+\phi_{i+1}}{\Delta x^{2}} .
$$

The original Savitzky-Golay filter [9] (or least-square smoothing filter) and its variants represents an important class of local methods for numerical differentiation of noisy data [10]. In their seminal paper, Savitzky and Golay demonstrated that fitting a polynomial to a set of input samples and then evaluating the obtained polynomial at a single point within the approximation interval is equivalent to discrete convolution with a fixed impulse response. The key derivation steps are summarized for a better appreciation of the proposed application to damage detection. A set of $(2 m+1)$ consecutive samples is considered together with a local coordinate system, i.e., $q \in\{-m, \ldots, 0, \ldots,+m\}$. The $l$ th-order least-square polynomial is represented by

$$
f(q)=\sum_{r=0}^{l} b_{r} q^{r}
$$

The Savitzky-Golay approach applies Eq. 2 at the midpoint only $(q=0)$ whereas the value of the output at the next sample is obtained by shifting the analysis interval to the right by one sample and repeating the procedure at the new midpoint. The sth-order derivative of Eq. 2 evaluated at $q=0$ only requires the expression for $b_{s}$. The central $s$ thorder derivative of the polynomial form in Eq. 2 can also be framed as:

$$
\phi^{s}(0)=\sum_{q=-m}^{m} h_{q}^{s} \phi_{q}
$$

in which $h_{q}^{s}$ is the convolution weight of the $q$ th filter point. Instead of considering a power series, the approach developed in [11] is based on the use of discrete orthogonal polynomials, whereby the Gram polynomials turn out to be particularly suitable to this case. In doing so, the sth-order derivative at any point $\xi$ is obtained by using the following expression:

$$
\phi^{s}(\xi)=\frac{1}{(\Delta x)^{s}} \sum_{q=-m}^{m} h_{q}^{\xi, s} \phi_{q}
$$

where the scale factor is needed when the original coordinate system is considered (e.g., the points are separated by $\Delta x \neq 1$ ). Convolution weight for $\phi_{q}$ with $-m \leq q \leq m$ has the form:

$$
h_{q}^{\xi, s}=\sum_{r=0}^{l} \frac{(2 r+1)(2 m)^{(r)}}{(2 m+r+1)^{(r+1)}} P_{r}^{m}(q) P_{r}^{m, s}(\xi),
$$

where

$$
P_{r}^{m}(\xi)=\sum_{a=0}^{r} \frac{(-1)^{a+r}(a+r)^{(2 a)}(m+\xi)^{(a)}}{(a !)^{2}(2 m)^{(a)}}
$$

and

$$
\begin{aligned}
P_{r}^{m, s}(q)= & \frac{2(2 r-1)}{r(2 m-r+1)}\left[q P_{r-1}^{m}(q)+s P_{r-1}^{m, s-1}(q)\right]- \\
& \frac{(r-1)(2 m+r)}{r(2 m-r+1)} P_{r-2}^{m, s}(q)
\end{aligned}
$$

are the $r$ th-order Gram polynomial and its $s$ th-order derivative, respectively. The calculation of the modal curvature through Eq. 4 is best performed by constructing the table $H^{s}(\xi, q)=\left\{h_{q}^{\xi, s}:-m \leq \xi \leq+m,-m \leq q \leq+m, s=2\right\}$ using Eq. 5. This strategy is computationally efficient because the convolution weights do not change, provided that the measurement points as well as $m$ and $l$ do not vary with the mode shape number in both undamaged and damaged situations. Note that the classical Savitzky-Golay approach [9] does not allow the calculation of the modal curvatures at the first $m$ points and at the last $m$ points. By using the Gram polynomial-based strategy developed in [11], the curvatures at the first $m$ points and at the last $m$ points are calculated by using the coefficients of $H^{s}(\xi, q)$ for $\xi$ from $-m$ to -1 and for $\xi$ from 1 to $m$, respectively. As in the classical Savitzky-Golay approach, the rest of the $n$ samples uses the center point weighting, which is obtained by setting $\xi=0$.

\section{Applications}

An extensive numerical study is conducted for aluminum and composite laminated plates. The Mindlin-Reissner plate model is considered wherein damage or defects are introduced as localized smoothed variations of the undamaged original configuration. The finite element software COMSOL Multiphysics is used to perform FE discretization of the equations of motion provided in PDE form. The composite plate has four layers with lay-up $0^{\circ} / 45^{\circ} / 90^{\circ} /$ $45^{\circ}$. The elastic constants are: $E_{1}=137.137 \mathrm{GPa}, E_{2}=9.308$ $\mathrm{GPa}, v_{12}=0.304, v_{21}=0.017, G_{12}=4.551 \mathrm{GPa}, G_{23}=4.206$ $\mathrm{GPa}$, and the mass per unit volume is $\rho=1568 \mathrm{~kg} / \mathrm{m}^{3}$. The damage is introduced by halving the stiffness of the second layer within a region having an extension equal to $4 \%$ of the plate side. A white Gaussian noise was added to the modal displacements in such a way that the final error (about $1 \%$ ) is able to simulate experimental data after denoising. Figure 1 shows a sample of the obtained numerical results for the composite laminated plate. This figure demonstrates that the central difference method does not lead to the proper identification of the damage because of countless false positives. On the other hand, use of the Savitzky-Golay filter gives a clear definite spike at the exact damage location.

Experiments were also performed on isotropic plate structures using SLV (see Fig. 2). The experimental results shown in Fig. 3 further confirm that the Savitzky-Golay filter provides a satisfactory identification of the damage in plate-like structures. Both location and extension of the damage are very well identified for the steel plate. The damage extension is identified properly for the aluminum plate whereas the location is slightly off from the exact one. 

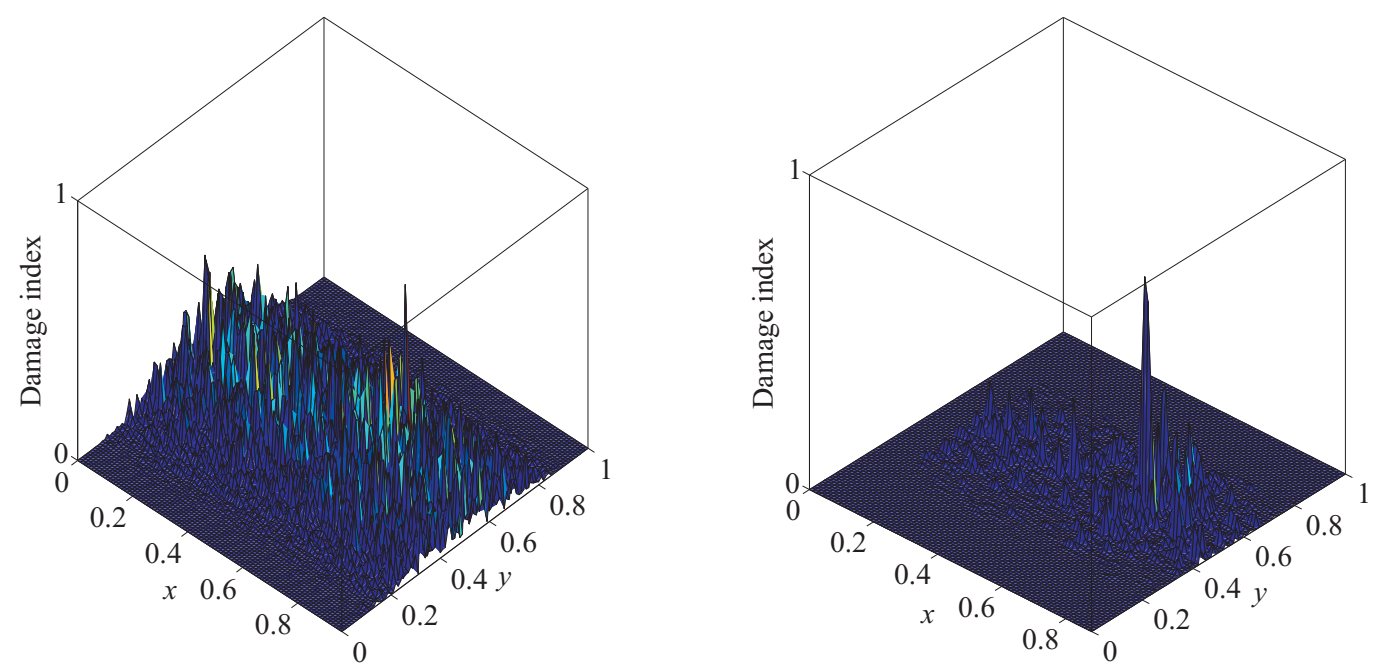

Fig. 1. Damage detection in composite laminated plate based on numerical data (the co-ordinates of the midpoint of the narrow damaged region are $x=0.67$ and $y=0.59$ ): comparison between central difference method (left) and Savitzky-Golay filter (right).
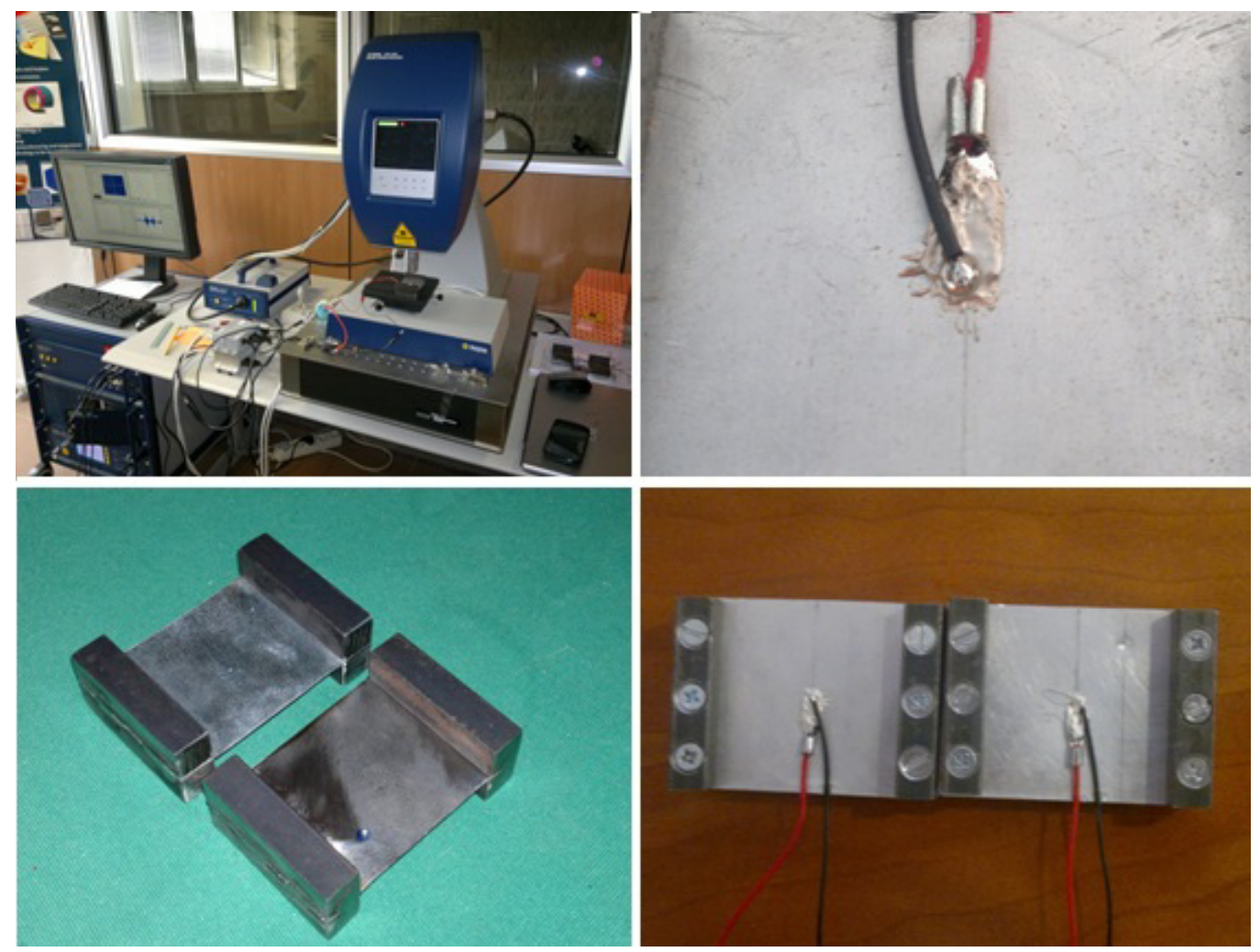

Fig. 2. Experimental layout (clockwise from the upper left corner): Polytec scanning laser vibrometer, piezoelectric actuator, undamaged and damaged $50 \mathrm{~mm} \times 50 \mathrm{~mm}$ aluminum and steel plates (thickness equal to $0.5 \mathrm{~mm}$ ). The damage is introduced by halving the plate thickness within a circle having $2 \mathrm{~mm}$ diameter and the center about $14 \mathrm{~mm}$ far from one corner.

\section{Conclusions}

Damage detection by means of modal curvatures computed via numerical differentiation has been investigated. Although the central difference method is largely employed, this study - in consonance with previous works - confirmed that it is not effective for damage detection (especially when considering relatively dense arrays of measurement points). As well known, keeping the measurement error as low as possible and reducing the noise are beneficial for damage detection, however, this does not solve the problem. On the contrary, numerical and experimental results demon- strate that the Savitzky-Golay filter allows a satisfactory identification of damage in plate-like structures. Despite its simplicity, this numerical technique leads to a remarkable reduction of the number of false alarms. A comprehensive assessment of the Savitzky-Golay filter and cubic smoothing spline in damage detection by modal curvatures is the topic of a forthcoming paper [12].

\section{Acknowledgments}

This work was partially supported by the Italian Ministry of Education, University and Scientific Research (PRIN 

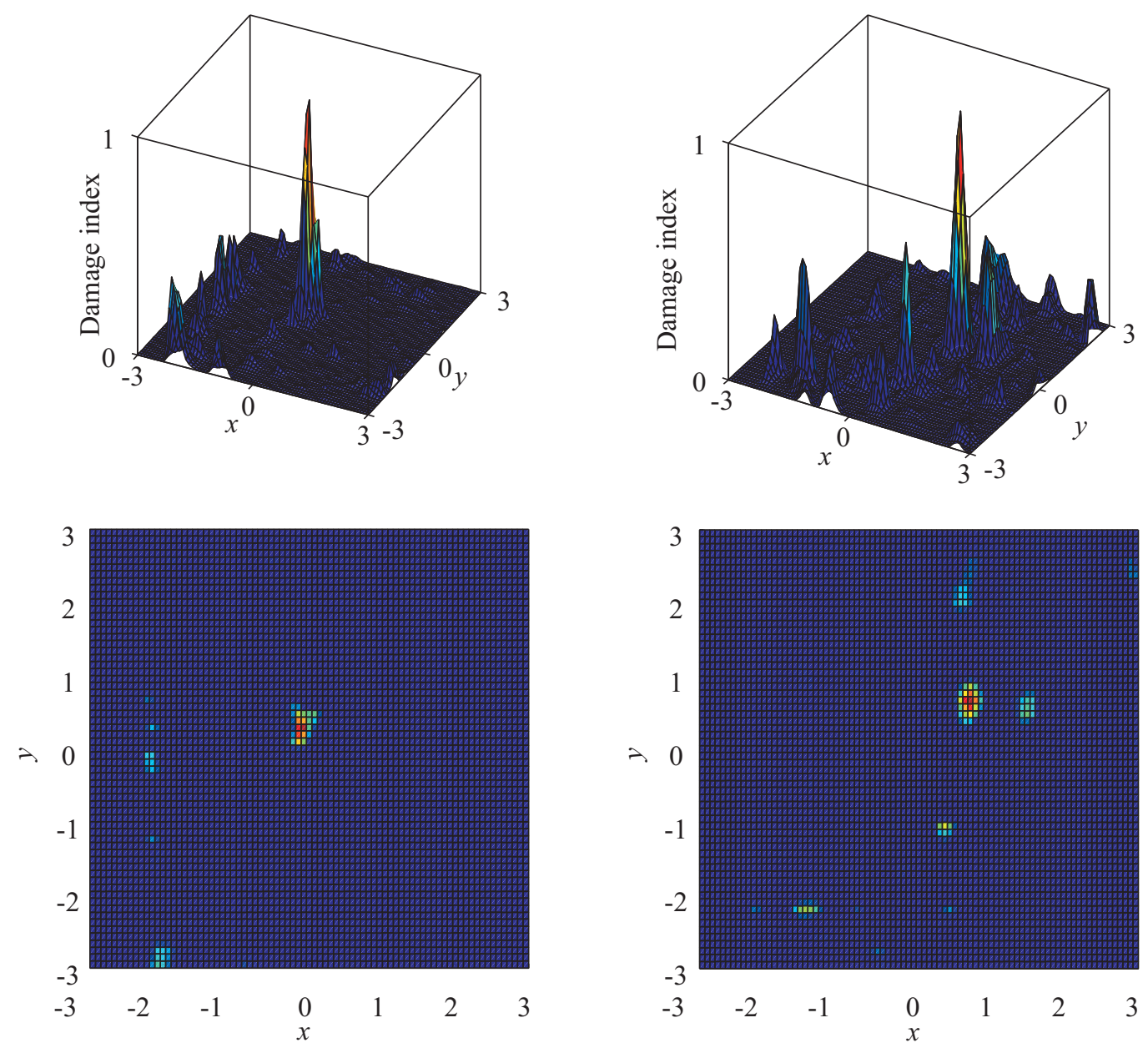

Fig. 3. Damage detection in isotropic plates based on experimental data (the curvatures are computed by the Savitzky-Golay filter over a $50 \times 50$ grid of points centered at the damaged region): steel plate (left column) and aluminum plate (right column).

Grant No. 2010BFXRHS-002). The work of Giuseppe Quaranta is framed within the research project "DPC-ReLUIS 2014, RS 4 - Osservatorio sismico delle strutture e monitoraggio".

\section{References}

1. A.K. Pandey, M. Biswas, and M.M. Samman, J. Sound Vib. 145, (1991), 321-332.

2. M.M. Abdel Wahab and G. De Roeck, J. Sound Vib. 226, (1999), 217-235.

3. A. Gentile and A. Messina, Int. J. Solids Struct. 40, (2003), 295-315.

4. M. Cao, W. Xu, W. Ostachowicz and Z. Su, J. Sound Vib. 333, (2014), 1543-1553.

5. H.M. Reis Lopes, J.V. Araújo dos Santos, C.M. Mota Soares, R.J. Miranda Guedes and M.A. Pires Vaz, Comput. Struct. 89, (2011), 1754-1770.

6. M. Cao and P. Qiao, Mech. Syst. Signal Pr. 23, (2009), 1223-1242.

7. P. Qiao, K. Lu, W. Lestari, and J. Wang, Compos. Struct. 80, (2007), 409-428.

8. A.P. Herman, A.C. Orifici, and A.P. Mouritz, Compos. Struct. 104, (2013), 34-42.

9. A. Savitzky and M. J. E. Golay, Anal. Chem. 36, (1964), 1627-1639.
10. K. Ahnert and M. Abel, Comput. Phys. Commun. 177, (2007), 764-774.

11. P.A. Gorry, Anal. Chem. 62, (1990), 570-573.

12. G. Quaranta, B. Carboni, W. Lacarbonara, J. Vib. Control, in press. 